\title{
HOXC9 directly regulates distinct sets of genes to coordinate diverse cellular processes during neuronal differentiation
}

\author{
Xiangwei Wang ${ }^{1 \dagger}$, Jeong-Hyeon Choi ${ }^{2,3+}$, Jane Ding ${ }^{2,4+}$, Liqun Yang ${ }^{6 \dagger}$, Lambert C Ngoka ${ }^{2}$, Eun J Lee ${ }^{2,5}$, \\ Yunhong Zha ${ }^{7}$, Ling Mao ${ }^{8}$, Bilian Jin ${ }^{2,5}$, Mingqiang Ren ${ }^{2,4}$, John Cowell ${ }^{2,4}$, Shuang Huang ${ }^{2,5}$, Huidong Shi ${ }^{2,5}$, \\ Hongjuan Cui ${ }^{6^{*}}$ and Han-Fei Ding ${ }^{2,4,5^{*}}$
}

\begin{abstract}
Background: Cellular differentiation is characterized by the acquisition of specialized structures and functions, cell cycle exit, and global attenuation of the DNA damage response. It is largely unknown how these diverse cellular events are coordinated at the molecular level during differentiation. We addressed this question in a model system of neuroblastoma cell differentiation induced by HOXC9.

Results: We conducted a genome-wide analysis of the HOXC9-induced neuronal differentiation program. Microarray gene expression profiling revealed that HOXC9-induced differentiation was associated with transcriptional regulation of 2,370 genes, characterized by global upregulation of neuronal genes and downregulation of cell cycle and DNA repair genes. Remarkably, genome-wide mapping by ChIP-seq demonstrated that HOXC9 bound to 40\% of these genes, including a large number of genes involved in neuronal differentiation, cell cycle progression and the DNA damage response. Moreover, we showed that HOXC9 interacted with the transcriptional repressor E2F6 and recruited it to the promoters of cell cycle genes for repressing their expression.
\end{abstract}

Conclusions: Our results demonstrate that HOXC9 coordinates diverse cellular processes associated with differentiation by directly activating and repressing the transcription of distinct sets of genes.

Keywords: Neuronal differentiation, Cell cycle arrest, DNA damage response, E2F6, HOXC9, Neuroblastoma

\section{Background}

Cellular differentiation is an essential process of normal development by which a stem or progenitor cell becomes a post-mitotic, specialized cell with unique morphology and function. In addition, it has long been recognized that differentiated cells of both normal and tumor origin are defective in the DNA damage response and repair at the global level, displaying a marked increase in sensitivity to ionizing radiation and other DNA damaging agents [1-3]. Consistent with these observations, recent studies have shown that brain and breast cancer stem cells, a small

\footnotetext{
* Correspondence: hcui@swu.edu.cn; hding@gru.edu

${ }^{\dagger}$ Equal contributors

${ }^{6}$ State Key Laboratory of Silkworm Genome Biology, Institute of Sericulture and System Biology, Southwest University, Chongqing, China

${ }^{2}$ Cancer Center, Georgia Regents University, Augusta, GA 30912, USA

Full list of author information is available at the end of the article
}

subpopulation of tumor cells thought to be responsible for initiating and sustaining tumor growth [4-6], are more resistant to irradiation and chemotherapy than bulk tumor cells [7-10]. Particularly interesting is the observation that inhibition of DNA damage checkpoint kinases can reverse the radioresistance of glioma stem cells [7]. Thus, a molecular understanding of cellular differentiation may suggest new therapeutic strategies that target both cell proliferation and the DNA damage response.

Among the genes that have a critical role in the control of cellular differentiation are the HOX gene family members. $H O X$ genes encode a family of transcription factors that function as master regulators of morphogenesis and cell fate specification [11-13]. Dysregulation of HOX gene expression has been implicated in the pathogenesis of cancers of different tissue types. In most tumor types, $H O X$ genes function as oncogenes to promote cancer development such as

\section{Ciomed Central}


HOXA9 in leukemia and HOXB13 in ovarian and breast cancers $[13,14]$. However, in neuroblastoma, a common childhood malignant tumor of the sympathetic nervous system $[15,16]$, there is evidence suggesting that HOX genes may function as tumor suppressors [13]. Particularly, downregulation of $\mathrm{HOXC9}$ expression is significantly associated with poor prognosis in neuroblastoma patients $[17,18]$.

Neuroblastoma cells can be induced to undergo neuronal differentiation by serum deprivation [19], nerve growth factor [20] or retinoic acid (RA) [21]. RA-induced neuronal differentiation of neuroblastoma cells is a well-established model for molecular investigation of neuronal differentiation [22]. We recently reported that RA-induced differentiation of neuroblastoma cells required the activation of several HOX genes [18,23]. Among them, HOXC9 appeared to be a major mediator of RA action in neuroblastoma cells. HOXC9 expression was upregulated by RA, and silencing HOXC9 expression conferred resistance to RA-induced differentiation. Importantly, ectopic HOXC9 expression alone was sufficient to induce growth arrest and morphologic differentiation in neuroblastoma cells, fully recapitulating the neuronal differentiation phenotype induced by RA [18].

Differentiated neuroblastoma cells morphologically and functionally resemble mature peripheral neurons characterized by G1 arrest, extensive neurite outgrowth, and significant resting potential. It has long been observed that differentiated neuroblastoma cells are highly sensitive to UV and X-ray radiation with a significantly reduced rate of DNA damage repair [20,24-27]. The molecular basis for the differentiation-induced radiosensitivity is not well understood. The biological functions of RA are mediated by multiple isotypes of RA receptors (RARs) and retinoid $\mathrm{X}$ receptors (RXRs), which form RAR/RXR heterodimers that bind RA response elements in the regulatory regions of RA target genes and regulate their transcription [28]. The complexity of multiple RARs and RXRs involved in the action of RA presents a daunting challenge to dissect the molecular mechanism that coordinates the diverse cellular events associated with differentiation. Thus, the finding that HOXC9 alone is able to initiate a robust transcriptional program that drives neuronal differentiation provides a unique experimental system for this investigation. In this study, we conducted genome-wide profiling of the HOXC9-initiated transcriptional program. Our investigation reveals that $\mathrm{HOXC} 9$ directly regulates the expression of three major sets of genes that separately control neuronal differentiation, cell cycle progression, and the DNA damage response.

\section{Results}

\section{Gene expression profiling of HOXC9-induced neuronal} differentiation

To gain a molecular understanding of HOXC9-induced differentiation, we conducted microarray gene expression profiling of human neuroblastoma $\mathrm{BE}(2)-\mathrm{C} / \mathrm{Tet}-\mathrm{Off} / \mathrm{myc}-$ HOXC9 cells, which express myc-tagged human HOXC9 and undergo neuronal differentiation in the absence of doxycycline [18] (Figure 1A). The profiling analysis identified a total of 2,370 genes that were differentially expressed $(\geq+1.5$ and $\leq-1.5$ fold, $P<0.01)$, with 879 genes being upregulated and 1,491 genes downregulated (Additional file 1: Table S1). Gene annotation enrichment analysis revealed that HOXC9-induced differentiation is characterized by a genome-wide coordination in transcriptional regulation of genes that control neuronal differentiation, cell cycle progression, and the DNA damage response.

\section{Global upregulation of neuronal genes}

Gene Ontology (GO) analysis of the 879 HOXC9upregulated genes by DAVID [29,30] revealed that they were significantly enriched for genes that control nervous system development such as neuron generation and differentiation, axonogenesis, and synapse formation and organization (Figure $1 \mathrm{~B}$ and Additional file 2: Table S2, enrichment fold $\geq 2.0$, false discovery rate (FDR) $\leq 1 \%$ ). A total of $105 \mathrm{HOXC} 9$-responsive genes were involved in nervous system development (Figure 1B), accounting for approximately $12 \%$ of the 879 genes upregulated by HOXC9. We obtained similar results with Gene Set Enrichment Analysis (GSEA), which showed significant enrichment of gene sets involved in synaptogenesis and neuron differentiation among the genes upregulated by HOXC9 (Figure 1C). Particularly significant was the activation of ASCL1, GFRA3, RET, and NTN3 (Figure 1D). ASCL1, a member of the basic helix-loop-helix (bHLH) family of transcription factors, is a master regulator in the generation and differentiation of sympathetic neurons [31,32]. GFRA3 encodes the glial cell line-derived neurotrophic factor (GDNF) family receptor alpha 3 (GFR $\alpha 3)$, which forms a receptor complex with RET that preferentially binds the GDNF family ligand Artemin. This receptor signaling has a critical role in embryonic development of the sympathetic nervous system, promoting the survival, differentiation, axonal outgrowth, and target innervation of sympathetic neurons [33]. NTN3 (netrin 3) belongs to a family of extracellular proteins that promote axon growth and migration during the development of the nervous system [34]. Ingenuity Pathways Analysis (IPA) further revealed a network of HOXC9-upregulated genes relevant to the development and function of sympathetic neurons (Additional file 3: Figure S1). Together, these analyses demonstrate that HOXC9 activates a large number of neuronal genes, providing the molecular mechanism for its ability to induce neuronal differentiation of neuroblastoma cells.

Global downregulation of cell cycle and DNA repair genes GO analysis of the 1,491 HOXC9-downregulated genes revealed that they were remarkably enriched for genes 


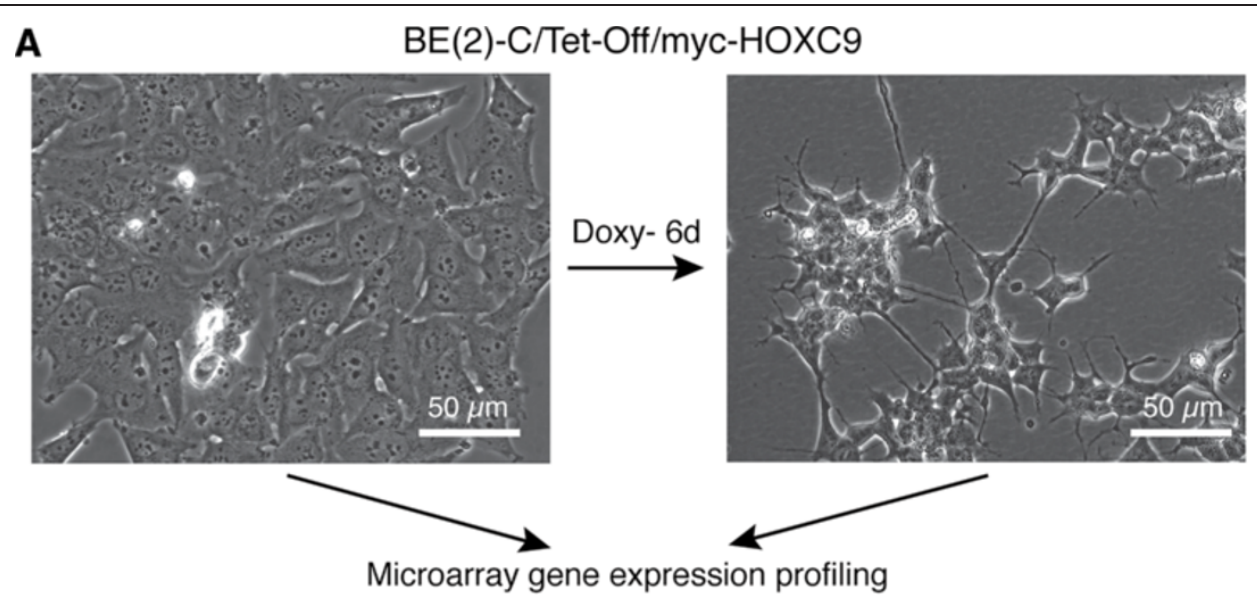

B

HOXC9-responsive genes_up

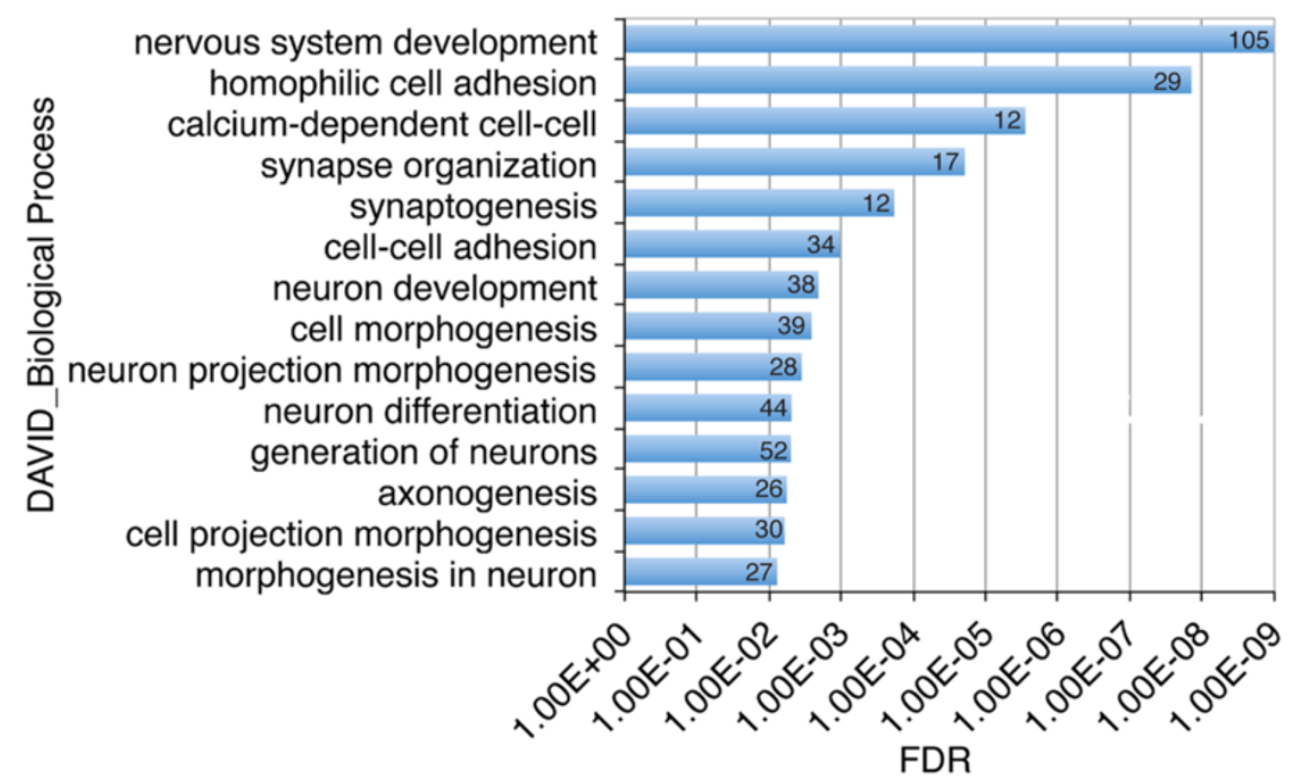

C

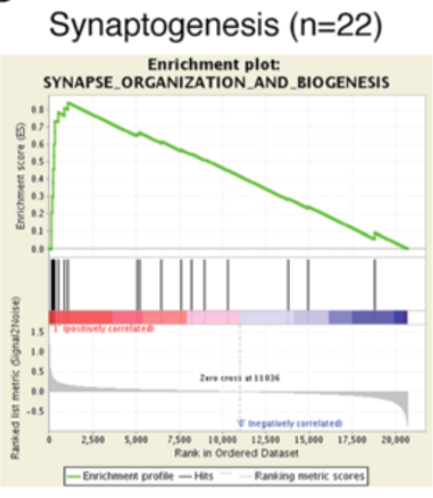

NOM $p=0 ; F D R<0.001$

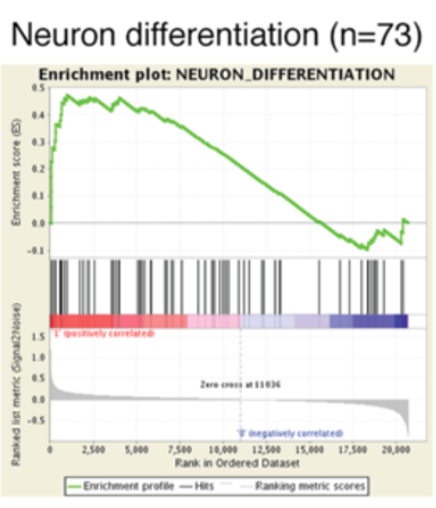

NOM $p=0.008 ; F D R=0.199$

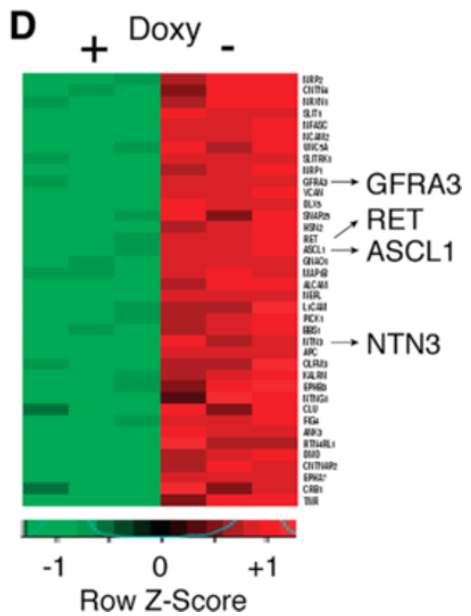

Figure 1 (See legend on next page.) 
that control cell cycle progression and the DNA damage response (Figure $2 \mathrm{~A}$ and Additional file 4: Table S3, enrichment fold $\geq 2.0$; FDR $\leq 1 \%$ ). The analysis identified 206 genes involved in cell cycle regulation and 98 genes in the DNA damage response (Figure 2A). Similarly, GSEA showed that among the genes downregulated by HOXC9, those regulating mitotic cell cycle, DNA replication and DNA repair were significantly enriched (Figure 2B). IPA further revealed that the downregulated genes include most of cyclin (CCN) and cyclin-dependent kinase $(\mathrm{CDK})$ genes, and genes that control DNA replication, mitosis, double-strand break (DSB) repair, base excision repair (BER), nucleotide excision repair (NER), mismatch repair (MMR), and Fanconi anemia (FA)-mediated repair (Additional file 3: Figures S2A-S2E and Additional file 4: Table S3). These findings suggest that global downregulation of cell cycle and DNA repair genes is the primary cause of the cell cycle arrest and attenuation of the DNA damage response associated with neuronal differentiation.

\section{Genome-wide mapping of HOXC9-binding sites}

We next asked how HOXC9 coordinates the expression of distinct sets of genes: the upregulation of genes critical for nervous system development and the downregulation of genes essential for cell cycle progression and the DNA damage response. Mechanistically, HOXC9 could function through a few master transcription factors, which in turn regulate their own subsets of target genes that work together to drive differentiation. Alternatively, HOXC9 could directly regulate distinct sets of genes to coordinate the cellular events associated with differentiation. To test these models, we conducted two independent anti-HOXC9 chromatin immunoprecipitation assays followed by massively parallel sequencing of the enriched DNA fragments (ChIP-seq) for genome-wide mapping of HOXC9-binding sites. We identified a total of 29,221 HOXC9-binding peaks with FDR less than 1\% (Figure 3A and Additional file 5: Table S4). Scatter plot analysis (Figure $3 \mathrm{~B}, \mathrm{R}=0.93$, correlation coefficient) and ChIP-seq tag profiles (Figure $3 \mathrm{C}$ ) demonstrated that the mapping data were highly reproducible between the two independent HOXC9 ChIP-seq samples. We next analyzed the distribution of HOXC9-binding peaks within the genome that was classified into functional categories including promoters (within $5 \mathrm{~kb}$ upstream of the transcription start site, TSS), 5'-untranslated regions (5'-UTRs), exons, introns, 3'-UTRs, downstream (within 5-kb downstream of the gene), and intergenic regions (outside $-5 \sim+5 \mathrm{~kb}$ of genes). The analysis revealed that a majority of HOXC9-binding peaks were localized in introns (41.2\%) and intergenic regions (43.4\%) (Figure 3D). However, after normalization to the size of these functional regions, it became clear that HOXC9-binding peaks were highly enriched in gene promoters and 5'-UTRs (Figure 3E and F). Analysis of the sequences covered by HOXC9-binding peaks with the motif-finding program MEME revealed that the most enriched binding motif (T/ATTTAT, E value $=1.6 \mathrm{e}-35)$ corresponds to the Drosophila Abd-B motif (MA0165.1, Figure 3G) and is highly homologous to the mouse Hoxc9-binding motif (ATTTAT) [35]. HOXC9 is a mammalian ortholog of the Drosophila Hox protein Abd-B. Thus, myc-tagged HOXC9 binds to cognate sequences in human neuroblastoma cells.

\section{Genome-wide identification of HOXC9 target genes}

The ChIP-seq assay revealed that a total of 4,992 genes contained at least one HOXC9-binding peak within 5-kb upstream or downstream of their genomic loci (Figure 3A and Additional file 6: Table S5). We next combined the anti-HOXC9 ChIP-seq data with the HOXC9 microarray data to generate a list of genes that were bound by HOXC9 and whose expression levels were significantly changed as a result of HOXC9 induction ( $\geq+1.5$ and $\leq-1.5$ fold, $P<0.01$ ). The analysis revealed that 954 genes or $40.3 \%$ of the 2,370 HOXC9-responsive genes are direct targets of HOXC9, with 445 and 509 genes being upregulated and downregulated, respectively (Additional file 7: Table S6). GO analysis of HOXC9 direct target genes revealed a transcriptional program characterized by coordinated regulation of genes critical for neuron differentiation, cell cycle progression, and the DNA damage response.

\section{HOXC9 directly induces a large number of neuronal genes}

The only sets of genes that were significantly enriched among the upregulated HOXC9 direct target genes are those exclusively involved in nervous system development, particularly the generation and differentiation of neurons and axonogenesis (Figure 4A and Additional file 8: Table S7, enrichment fold $\geq 2.0$, FDR $\leq 5 \%$ ). The 57 HOXC9 direct target genes account for $54.3 \%$ (57/105) of the HOXC9-responsive genes involved in nervous system development (Figure 1B). Among them are ASCL1, 


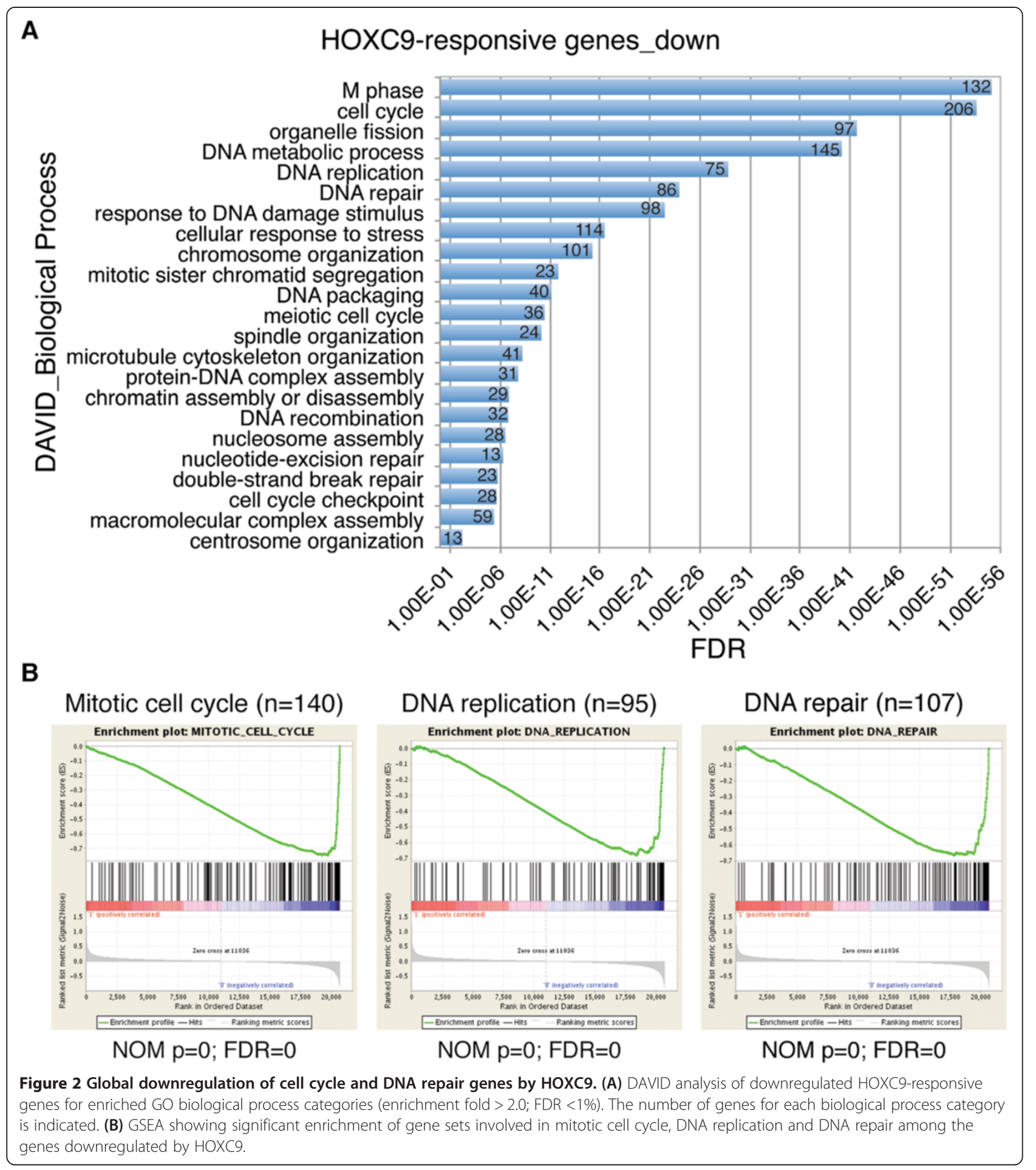

GFRA3, RET, and NTN3. Figure 4B shows the ChIPseq tag profiles of $\mathrm{HOXC} 9$ binding to the promoter regions of GFRA3, RET, and NTN3. As discussed above, these genes have a critical role in sympathetic neurogenesis and axonogenesis.
HOXC9 directly represses a large number of genes essential for cell cycle progression and the DNA damage response

GO analysis of the downregulated HOXC9 direct target genes revealed that they were significantly enriched for 


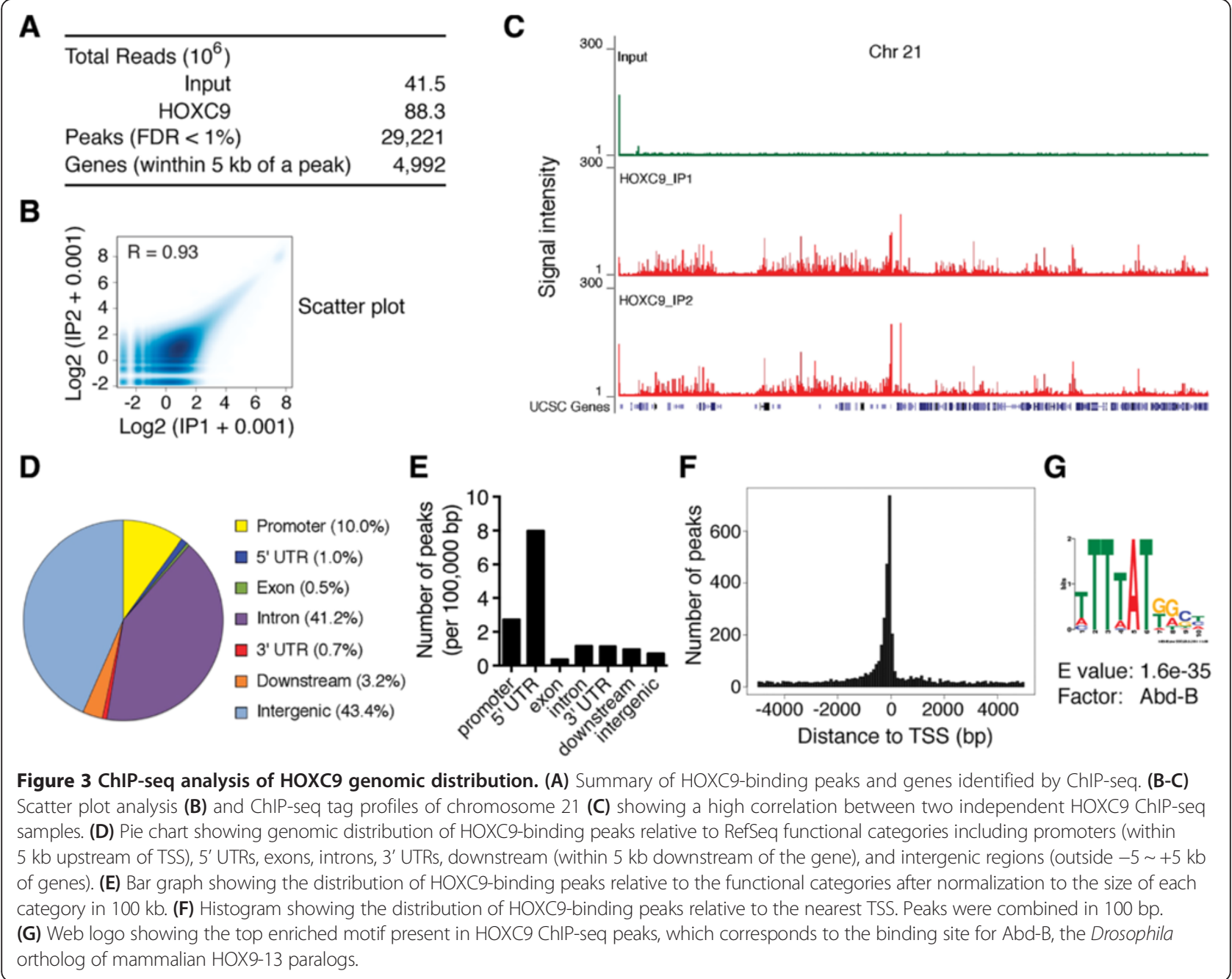

genes that control cell cycle progression and the DNA damage response (Figure 5A and Additional file 9: Table S8), enrichment fold $\geq 2.0, \mathrm{FDR} \leq 1 \%$ ). The analysis identified 52 cell cycle genes that were directly repressed by HOXC9 (Figure 5A), accounting for $25.2 \%(52 / 206)$ of the HOXC9-responsive genes involved in cell cycle regulation (Figure $2 \mathrm{~A}$ ). It was particularly striking that the vast majority of the HOXC9-repressed cell cycle genes are involved in the control of the $M$ phase $(n=25)$ and DNA replication $(n=21)$ (Figure $5 \mathrm{~A})$. Figure $5 \mathrm{~B}$ and $\mathrm{C}$ show the association of HOXC9 with the promoter regions of representative cell cycle genes, including $C D C 45 L$ and MCM3 (DNA replication), and CCNB1 and CDCA8 (M phase). CDC45L and MCM3 are components of the replicative complex that catalyzes DNA replication during the $\mathrm{S}$ phase [36], while CDCA8, also known as BOREALIN, is a component of the chromosomal passenger complex essential for mitosis and cell division [37].

We also identified 32 genes associated with the DNA damage response that were directly repressed by HOXC9
(Figure 5A), accounting for $32.7 \%$ (32/98) of the HOXC9-responsive genes involved in the DNA damage response. Figure 5D shows the binding of HOXC9 to the promoter of FANCM and to both the promoter and 3 ' region of FEN1. FANCM is a component of the FANCM-FAAP24-MHF protein complex that binds to DNA with interstrand cross-links and is responsible for recruiting the FA core complex to the damaged site [38]. FEN1 (flap endonuclease 1) is essential for DNA replication and repair by removing RNA and DNA 5 ' flaps [39].

Collectively, these findings suggest that HOXC9 directly regulates the expression of distinct sets of genes to coordinate the molecular and cellular processes characteristic of neuronal differentiation.

\section{HOXC9 targets E2F6 to the promoters of cell cycle genes}

We next sought to determine the molecular basis for HOXC9 regulation of gene expression by identifying HOXC9-interacting proteins. We used a myc-tag antibody to isolate myc-HOXC9 and its associated proteins from 


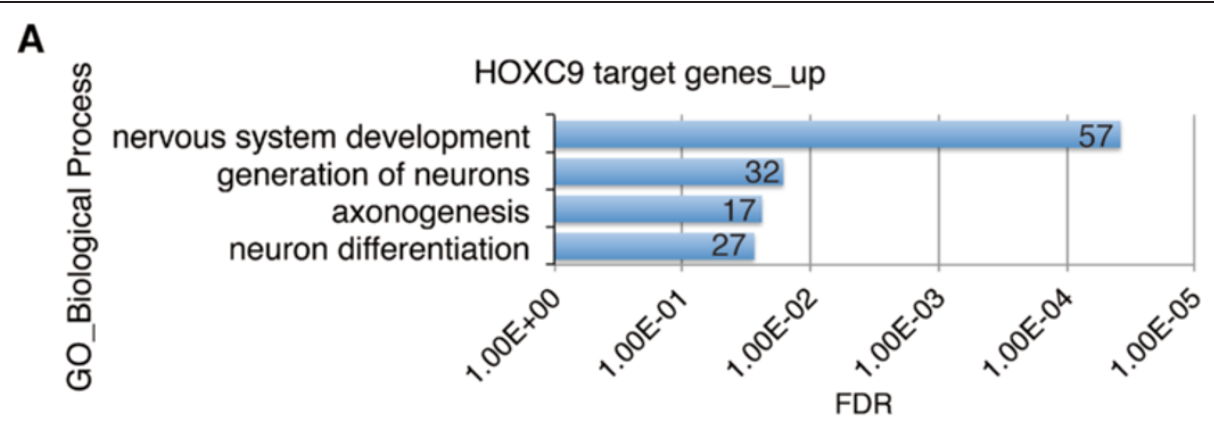

B

HOXC9 target genes_up_neuron differentiation
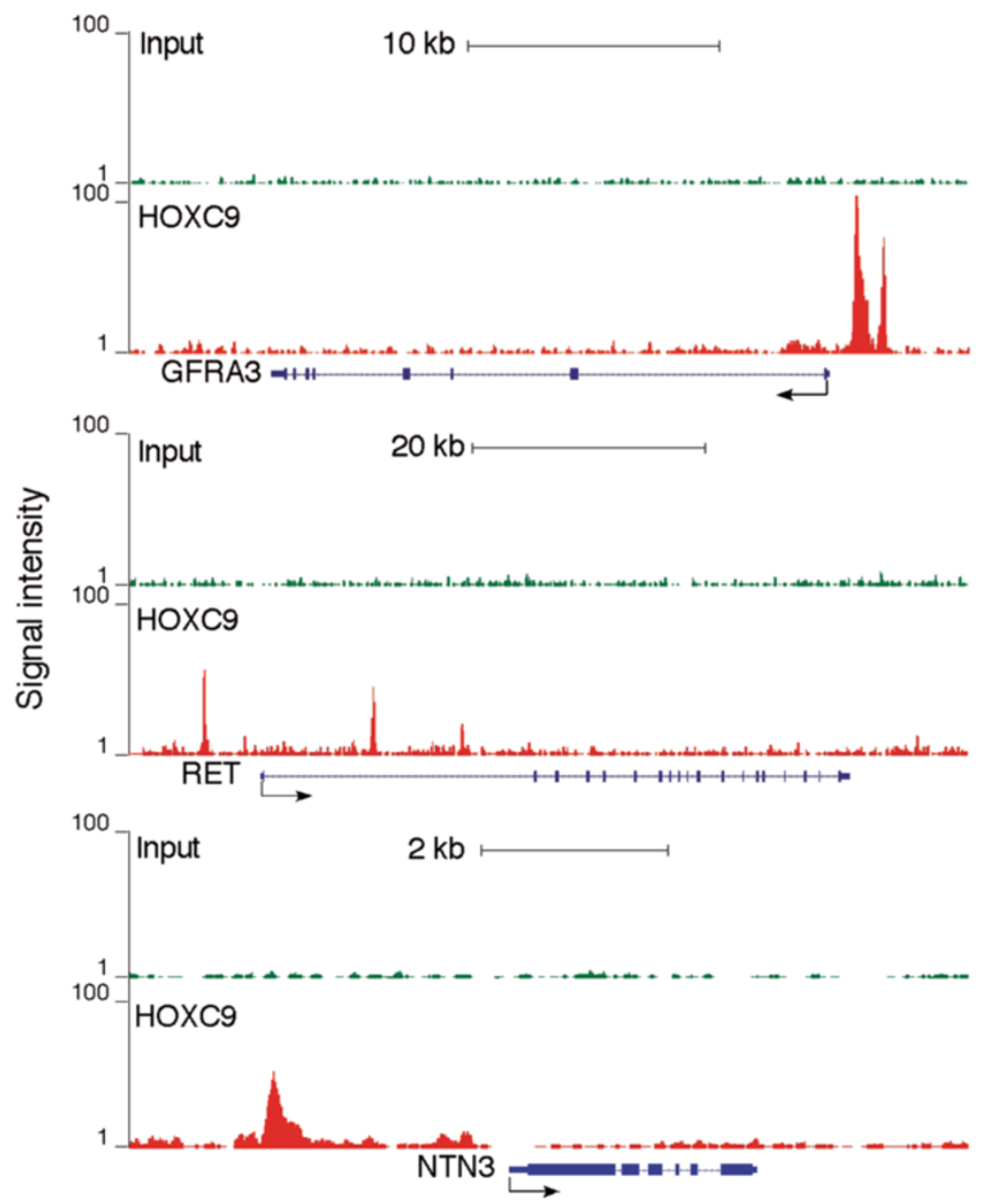

Figure 4 HOXC9 directly induces a large number of neuronal genes. (A) DAVID analysis of upregulated HOXC9 target genes for enriched GO biological process categories (enrichment fold $>2.0$; FDR $<5 \%$ ). The number of genes for each biological process category is indicated. (B) ChIP-seq tag profiles showing HOXC9 binding to representative upregulated HOXC9 target genes involved in nervous system development (GFRA3, top; RET, middle; NTN3, bottom).

nuclear extracts of BE(2)-C/Tet-Off/myc-HOXC9 cells cultured in the absence of doxycycline for 6 days (Additional file 3: Figure S3A). Mass spectrometric analysis of two independent samples identified E2F6 as a HOXC9- interacting protein (Additional file 3: Figure S3B), a well characterized transcriptional repressor that plays a major role in repressing E2F-responsive genes essential for cell proliferation [40]. It is known that E2F family proteins 


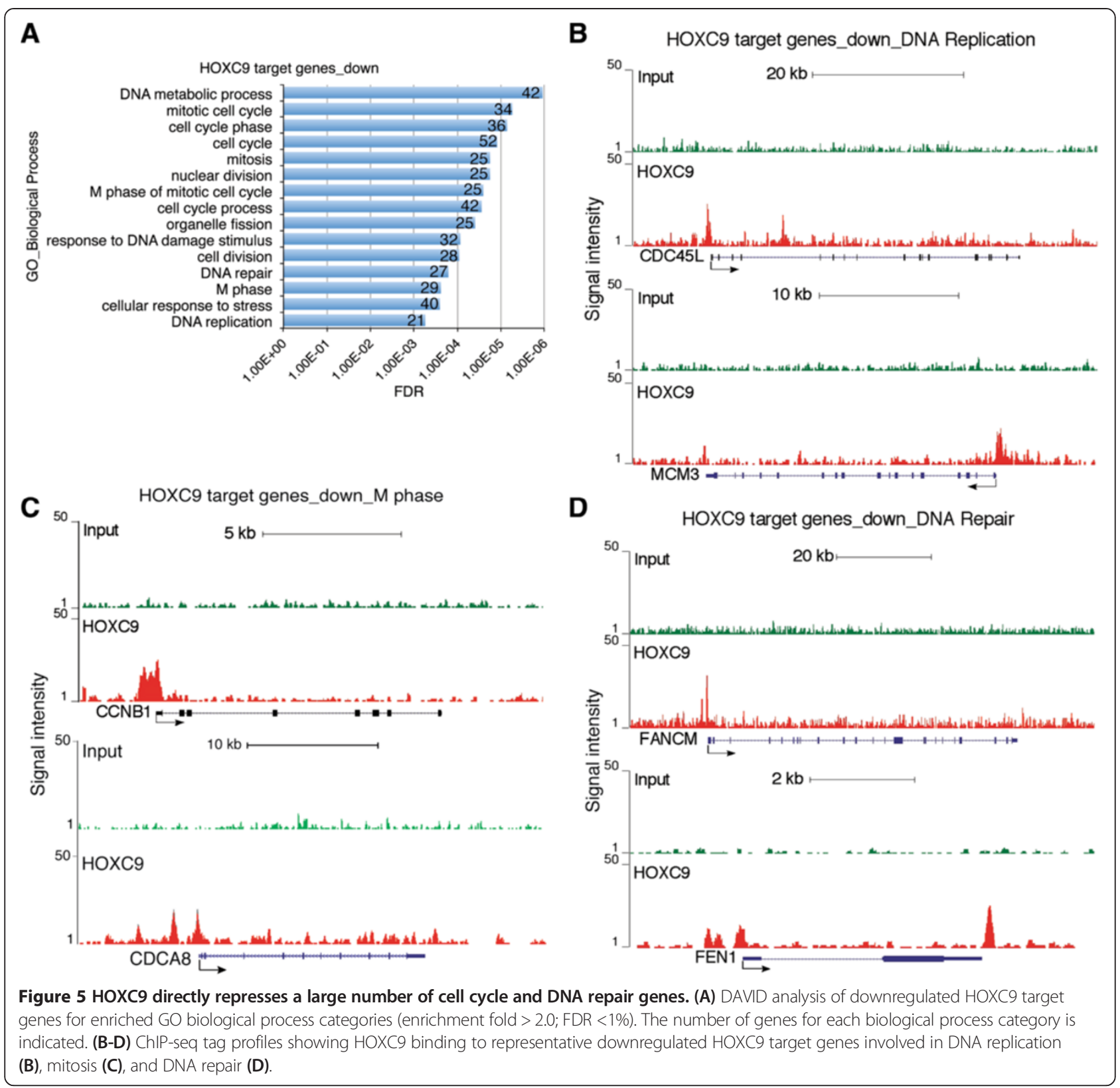

(E2F1-6) share the same core consensus G/CTTTG/C binding site [41]. Interestingly, GSEA revealed significant enrichment of the E2F-binding motif among the genes downregulated by HOXC9 (Additional file 3: Figure S3C). Taken together, these observations suggest that E2F6 has an important role in HOXC9-mediated repression of cell cycle genes.

To corroborate the finding of mass spectrometry, we performed co-immunoprecipitation (Co-IP) experiments using nuclear extracts from $\mathrm{BE}(2)-\mathrm{C} / \mathrm{Tet}-\mathrm{Off} /$ myc-HOXC9 cells cultured in the absence of doxycycline for 6 days. The myc-tag antibody, but not control IgG, precipitated myc-HOXC9 and E2F6 (Figure 6A, left panel). Reciprocally, an E2F6 antibody precipitated E2F6 and myc-HOXC9 (Figure 6A, right panel). We next performed size-exclusion chromatography using the same nuclear extracts. Immunoblot analysis revealed the presence of HOXC9 $(\sim 31 \mathrm{kDa})$ in two complexes: the larger complex (peak at fraction 20) had an estimated molecular mass of $\sim 1,800 \mathrm{kDa}$ and the other (peak at fraction 34) of $\sim 250 \mathrm{kDa}$ (Figure 6B). A significant amount of endogenous E2F6 $(\sim 36 \mathrm{kDa})$ co-eluted with the 1,800-kDa HOXC9 complex, whereas MEIS2 $(\sim 37-49 \mathrm{kDa})$, which interacts with HOX proteins and functions as a HOX cofactor [12], exclusively co-eluted with the $250 \mathrm{kDa}-\mathrm{HOXC} 9$ complex (Figure 6B). Co-IP experiments using pooled fractions confirmed the association 

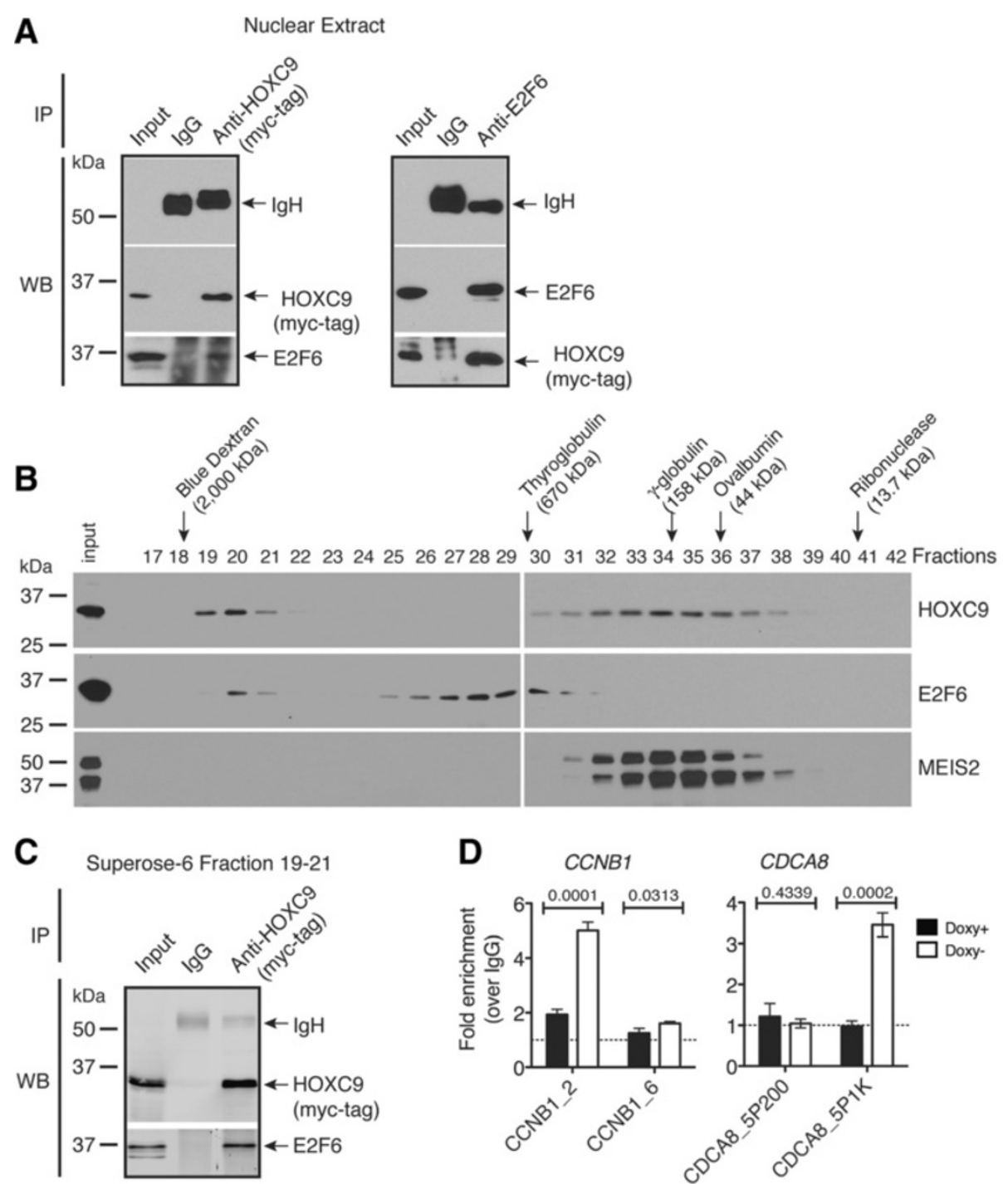

Figure 6 HOXC9 interacts with E2F6 and recruits it to cell cycle genes. (A) Reciprocal Co-IP of myc-HOXC9 and E2F6 in nuclear extracts from BE(2)-C/Tet-Off/myc-HOXC9 cells cultured in the absence of doxycycline (Doxy) for 6 days. (B) Size-exclusion chromatography analysis of complexes containing myc-HOXC9, E2F6 or MEIS2 in nuclear extracts of BE(2)-C/Tet-Off/myc-HOXC9 cells cultured in the absence of Doxy for 6 days. (C) Co-IP of myc-HOXC9 and E2F6 in pooled Superose-6 fractions 19-21. (D) ChIP-qPCR analysis showing E2F6 binding to specific promoter regions of the cell cycle genes CCNB1 and CDCA8 in BE(2)-C/Tet-Off/myc-HOXC9 cells before (Doxy+) and after (Doxy-) HOXC9 induction. Dashed lines indicate lgG control. Error bars represent SD $(n=3)$. Data were analyzed with unpaired, two-tailed Student's $t$-test and $p$ values are indicated.

of HOXC9 with E2F6 within the larger complex (Figure 6C).

To determine whether the HOXC9-E2F6 interaction plays a role in recruiting E2F6 to HOXC9 target genes in vivo, we performed anti-E2F6 ChIP using $\mathrm{BE}(2)-\mathrm{C} /$ Tet-Off/myc-HOXC9 cells before and after HOXC9 induction. HOXC9 induction had no apparent effect on E2F6 expression as determined by microarray gene expression profiling (-1.003 fold). ChIP-qPCR assay revealed that E2F6 was recruited to specific promoter regions of the cell cycle genes CCNB1 and CDCA8 only after HOXC9 induction (Figure 6D). By contrast, no significant binding of E2F6 to the NEFM promoter was observed before and after HOXC9 induction (Additional file 3: Figure S4A). As reported previously, NEFM is a neuronal gene directly activated by HOXC9 during differentiation [18] (See also Additional file 3: Figure S4B). Together, these data suggest that elevated levels of HOXC9 facilitate the formation of a repressive complex with E2F6, which is then recruited to cell cycle but not neuronal genes during differentiation.

\section{E2F6 is essential for HOXC9-induced cell cycle arrest and transcriptional repression of cell cycle genes}

To determine the functional significance of the HOXC9E2F6 interaction, we examined the effect of E2F6 knockdown on HOXC9-induced growth arrest. We 
depleted E2F6 using short hairpin RNA (shRNA) sequences targeting different coding regions of the human E2F6 gene (Figure 7A). Cells with E2F6 knockdown were highly resistant to HOXC9-induced G1 arrest, showing continued cell proliferation (Figure 7B) and cell cycle progression (Figure 7C) following HOXC9 induction. This was accompanied by a marked decrease in the population of cells in the G1 phase and a significant increase in the population of cells in the $S$ phase (Figure 7D). In addition, E2F6 knockdown largely abrogated the ability of HOXC9 to repress cyclin A2 and B1 expression, but had no significant effect on HOXC9 induction of NEFM (Figure 7E and F), a finding consistent with the observation of no significant E2F6 binding to the NEFM promoter during HOXC9induced differentiation (Additional file 3: Figure S4A). Together, these findings identify an essential and specific role for E2F6 in HOXC9 induction of growth arrest and repression of cell cycle genes.

\section{Discussion}

In this report, we present evidence for a master regulator of development with the capacity to coordinate diverse cellular events characteristic of neuronal differentiation by simultaneously and directly regulating distinct sets of genes (Figure 8). Through gene expression profiling, we show that HOXC9-induced neuronal differentiation is characterized at the molecular level by transcriptional regulation of 2,370 genes, with global activation of genes that promote nervous system development and repression of genes that are essential for cell cycle progression and the DNA damage response. Moreover, through a combination of genome-wide mapping of HOXC9 binding sites and gene expression profiling, we show that HOXC9 directly regulates the expression of 954 genes, $\sim 40 \%$ of the 2,370 HOXC9-responsive genes, including a large number of genes required for neuronal differentiation, cell cycle progression and the DNA damage response. Finally, we identify an essential role for E2F6 in HOXC9 repression of cell cycle genes and induction of G1 arrest.

Our findings that $\mathrm{HOXC} 9$ can both activate and repress gene transcription are consistent with previous observations from the study of spinal cord development in chick and mouse embryos. In the developing spinal cord, Hoxc9 functions as a transcription activator to promote the fate of preganglionic motor column (PGC) neurons [42], most likely through its interaction with the transcription factor FoxP1 [43,44]. However, Hoxc9 can also specify the fate of hypaxial motor column (HMC) neurons by repressing the Hox genes that promote the switch of HMC neurons to the lateral motor column (LMC) neurons [35]. Importantly, our study further demonstrated that within the same population of neuroblastoma cells, HOXC9 could simultaneously activate the genes that promote neuronal differentiation and repress the genes that are essential for cell cycle progression and the DNA damage response. While the molecular basis for the transcription activator function of HOXC9 in neuroblastoma cells remain to be defined, we showed that the ability of HOXC9 to repress cell cycle genes depended on its interaction with the transcription repressor E2F6, a member of the E2F family of transcription factors that have a critical role in the control of cell proliferation [40].

Cellular differentiation is tightly linked to cell cycle exit, with the differentiated cell containing the G1 content of DNA. The molecular mechanism that couples cell cycle exit and differentiation is not well understood, although it is generally recognized that cell cycle regulators influence differentiation, and cell fate determinants influence the cell cycle [45-48]. A primary example is the CDK inhibitor $\mathrm{p} 27^{\mathrm{Kip} 1}$ as a key regulator that links cell cycle exit and differentiation during development. p2 $7^{\text {Kip1 }}$ induces G1 arrest by associating with CDK/cyclin complexes and inhibits their kinase activity [49]. Overexpression of $\mathrm{p} 27^{\mathrm{Xic} 1}$, a Xenopus homolog of $\mathrm{p} 27^{\mathrm{Kip} 1}$, in Xenopus retina glial progenitor cells promotes both cell cycle exit and differentiation [50]. Knockout and overexpression studies also demonstrate an important role of $\mathrm{p} 27^{\mathrm{Kip} 1}$ in neuronal differentiation in the mouse cerebral cortex by stabilizing Neurogenin 2 [51], a proneural bHLH transcription factor with a central role in cortical neurogenesis [52]. On the other hand, cell fate determinants can also modulate the expression of $\mathrm{p} 27^{\mathrm{Kip} 1}$ for coordinated regulation of cell cycle exit and differentiation. For instance, Drosophila proneural bHLH proteins cooperate with epidermal growth factor signaling to directly activate the transcription of Dapaco, a homolog of $\mathrm{p} 21^{\mathrm{Cip}} / \mathrm{p} 27^{\mathrm{Kip} 1}$, during the differentiation of photoreceptor cells [53].

Our findings suggest an alternative mechanism for coupling cell cycle exit and differentiation. HOXC9 does not regulate the expression of CDK inhibitors, including $\mathrm{p} 27^{\mathrm{Kip} 1}$ and $\mathrm{p} 21^{\mathrm{Cip}}$, and overexpression of either $\mathrm{p} 27^{\mathrm{Kip} 1}$ or $\mathrm{p} 21^{\text {Cip }}$ fails to stop the proliferation of $\mathrm{BE}(2)-\mathrm{C}$ cells [18]. Rather, HOXC9 induces G1 arrest by directly repressing a large number of genes essential for cell cycle progression through the $\mathrm{S}$ to $\mathrm{M}$ phases, including cyclin B1, CDCA3, CDCA8, BUB1B, MCM3 and MCM8. This transcriptional repression function of $\mathrm{HOXC} 9$ requires E2F6. We found that HOXC9 interacts with E2F6 and recruits it specifically to the promoters of cell cycle genes. E2F6 lacks a transactivation domain and functions as a transcriptional repressor for E2F-responsive genes that drive cell proliferation [54-58]. Mechanistically, E2F6 interacts with chromatin modifiers with transcription repressor activity to establish a repressive chromatin structure. These chromatin modifiers include the DNA methyltransferase Dnmt3b [59] and polycomb-group (PcG) proteins [60-63]. In our study, we identified HOXC9 and E2F6 within a complex of approximately $1,800 \mathrm{kDa}$. Whether this 


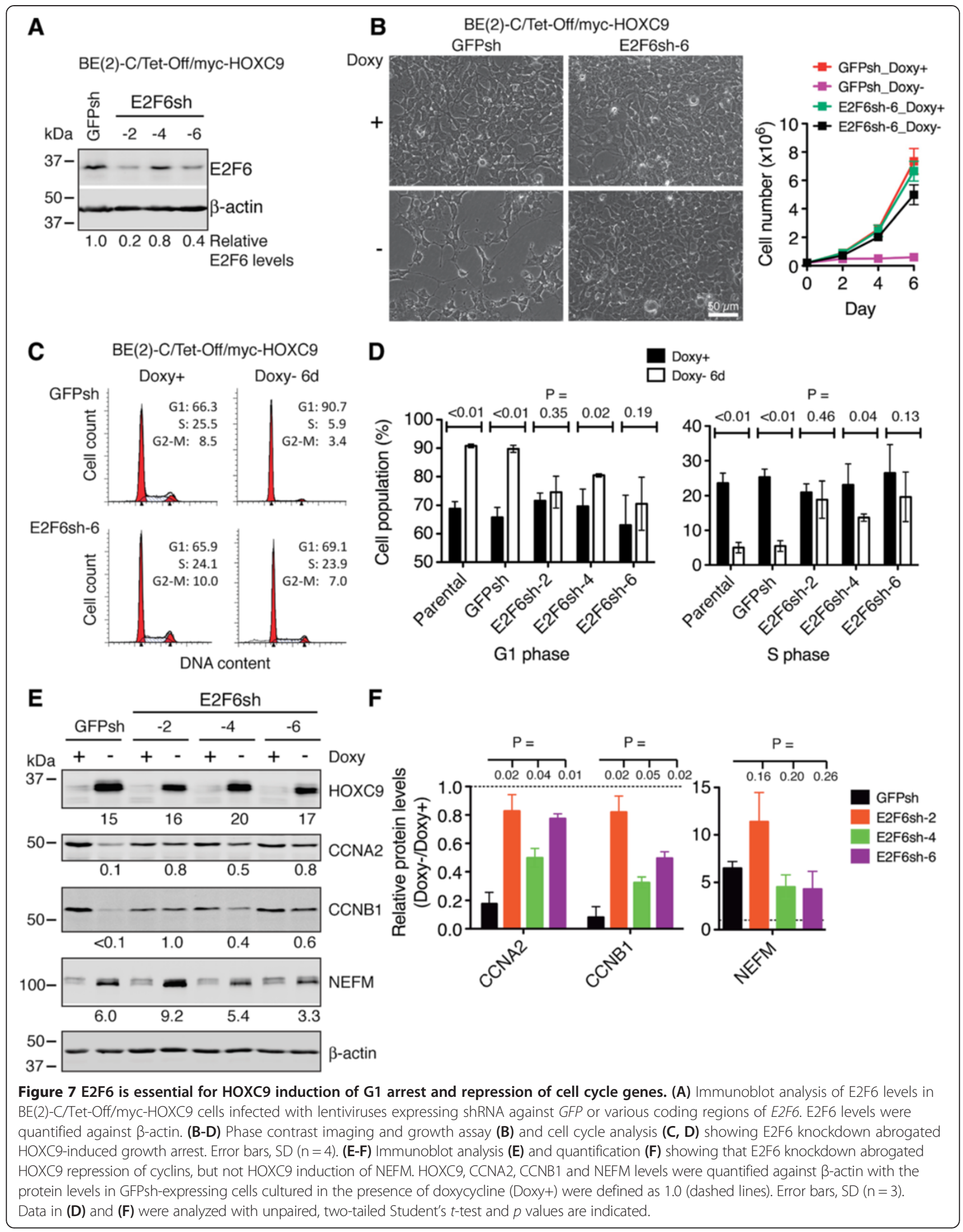




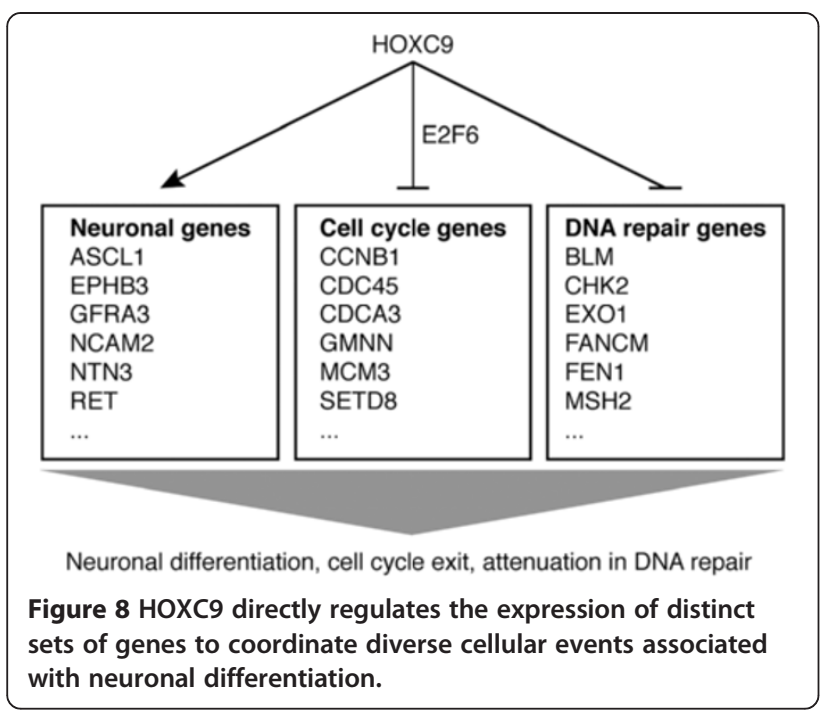

complex contains chromatin modifiers is currently under investigation.

Terminal cell differentiation is also tightly associated with a global reduction in DNA damage repair activities [1-3]. The underlying molecular mechanism is not well understood. It has been reported that E1 ubiquitinactivating enzyme can complement nucleotide-excision repair deficiency in extracts from differentiated macrophages, suggesting a role of ubiquitination in the control of the DNA damage response during differentiation [64]. Our study revealed that in HOXC9-induced neuronal differentiation, attenuation of the DNA damage response resulted from global transcriptional repression of DNA repair genes. This finding provides a molecular mechanism for the long observed differentiation-induced radiosensitivity in neuroblastoma cells [20,24-27]. For HOXC9-induced differentiation, a total of 98 genes with functions in the DNA damage response were significantly downregulated. These genes are involved in all types of DNA damage checkpoints and repair pathways. Importantly, we show that 32 of the 98 genes are direct targets of HOXC9. Thus, to a large extent, HOXC9 coordinates neuronal differentiation and attenuation of DNA repair activities by simultaneously activating neuronal genes and repressing DNA repair genes. Since the DNA damage response and DNA replication machineries share many components, we speculate that the downregulation of DNA repair genes during differentiation is a consequence of repression of cell cycle genes, particularly those involved in DNA replication.

The stem cell model of cancer attributes cancer growth to a subpopulation of cancer stem cells. It has been shown recently that cancer stem cells are intrinsically resistant to ionizing radiation and chemotherapy, as a result of enhanced checkpoint activation and more effective DNA damage repair [7-10]. Since differentiation is associated with global downregulation of DNA repair activities, a combination of differentiation-inducing agents and irradiation or chemotherapy may prove to be a more effective therapeutic strategy for targeting cancer stem cells.

\section{Conclusions}

Using neuroblastoma cell differentiation as an experimental system, we delineate a molecular mechanism by which HOXC9 coordinates diverse cellular processes associated with differentiation by directly activating and repressing the transcription of distinct sets of genes.

\section{Methods}

\section{Cell culture and growth assays}

The human neuroblastoma cell line BE(2)-C (CRL-2268, ATCC) with Tet-Off inducible expression of myc-tagged human HOXC9 has been described previously [18]. For E2F6 knockdown, BE(2)-C/Tet-Off/myc-HOXC9 cells were infected with lentiviruses expressing shRNA against E2F6 (TRCN013819, E2F6sh-2, TTTCGAGTTAAATAAACCAGC; TRCN013821, E2F6sh-4, ATTG GTGATGTCATACACTCT; TRCN018201, E2F6sh-6, ATCCAAAGCATCTTCCATTGC; Thermo Fisher Scientific). Cells were cultured in a 1:1 mixture of DMEM and Ham's nutrient mixture F12 supplemented with 10\% fetal bovine serum (Invitrogen-Gibco) in the presence or absence of doxycycline. Cells were examined and phase contrast images captured using an Axio Observer microscope and AxioVision software (Carl Zeiss MicroImaging), and viable cell numbers were determined by trypan blue exclusion assay. For cell cycle analysis, cells were fixed in $70 \%$ ethanol, incubated with ribonuclease A (Sigma-Aldrich), and stained with $20 \mu \mathrm{g} / \mathrm{ml}$ propidium iodide (Invitrogen-Gibco). Samples were analyzed using a FACSCalibur system and ModFitLT V3.2.1 software (BD Bioscience).

\section{Microarray gene expression profiling}

Total RNA was isolated using Trizol (Invitrogen) from three independent samples of $\mathrm{BE}(2)-\mathrm{C} /$ Tet-Off/myc-HOXC9 cells cultured in the presence or absence of doxycycline for 6 days. RNA was measured and quality assessed by a NanoDrop spectrophotometer and an Agilent 2100 Bioanalyzer (Agilent Technologies). Affymetrix microarray analysis was performed using the Human Gene 1.0 ST microarray chip. Data were normalized, significance determined by ANOVA, and fold change calculated with the Partek Genomics Suite (Partek Inc.). Gene annotation enrichment analysis was performed with DAVID v6.7 [30], GSEA [65], and IPA (Ingenuity ${ }^{\circ}$ Systems www.ingenuity.com) for all significantly changed genes $(\geq+1.5$ and $\leq-1.5$ fold, $P<0.01)$. 


\section{ChIP-seq and ChIP-qPCR}

Two independent preparations of BE(2)-C/Tet-Off/ myc-HOXC9 cells cultured in the presence or absence of doxycycline for 6 days were used for ChIP. Cross-linked chromatin DNA was sheared through sonication and immunoprecipitated using mouse anti-myc tag (clone 4A6, Millipore) or mouse anti-E2F6 (sc-53273, Santa Cruz Biotechnologies) according to the published procedure [66]. For ChIP-seq, libraries were generated from ChIP genomic DNA samples according to the Illumina ChIP-seq library construction procedure, and sequenced using Illumina Genome Analyzer IIx with a read length of 36 or 76 bp. For ChIP-qPCR, ChIP genomic DNA samples were assayed in triplicate by PCR using an iQ5 real-time PCR system (Bio-Rad) and the following primer sets that cover the promoter regions of CCNB1 (CCNB1_2 and CCNB1_6), CDCA8 (CDCA8_5P200 and CDCA8_5P1K), and NEFM (NEFM_5P1 and NEFM_5P2): CCNB1_2: CCAGAGAGTTGTTGCAACGAT, CTGGAGAGCAGT GAAGCCAGT; CCNB1_6: GGAAGGATTGATCAAACC CAG, AGTCACGGATCCGAAAGAAGG; CDCA8_5P200: GGTATTGCAGAGCCGCCA, CCTCCCCACCAACCCAC C; CDCA8_5P1K: TGGTGCCCATCAGGAGCC, GGCT ATGGGAGTGATAATC; NEFM_5P1: GCAGAAAGTAA TAAGCAACAA, CCTGCCTTCTGTAAAGTATTG; NEFM_ 5P2: CCTTTCCTGATTACTTACTGA, AGGGACTCCAGA CCGAAATAG.

\section{ChIP-seq data analysis}

Raw Illumina sequencing reads from the two independent ChIP replicates (rep1, GEO GSM848788 and rep2, GEO GSM848789) in the FASTQ format were cleaned using in-house scripts by trimming sequencing adaptors and low quality bases in both ends $(\mathrm{Q}<67$ in Illumina 1.5). Cleaned sequences were then mapped to the human genome (hg19) using Novoalign v2.07 for identifying the reads that were mapped uniquely to a single genomic locus. The identified reads from the rep1 ChIP sample (GEO GSM848788) were used for peak calling with Model-based Analysis of ChIP-Seq (MACS v1.4) [67], and only those peaks with FDR $<1 \%$ were compared with RefSeq genes in the UCSC genome browser and classified into functional categories such as promoters, 5'-UTRs, exons, introns, 3'-UTRs, downstream, and intergenic regions. To measure the correlation of two HOXC9 replicates, we used $200 \mathrm{bp}$ non-overlapping windows where a tag density is defined as the number of reads in a window. We calculated Pearson correlation coefficient with $\mathrm{R}>0.9$ being highly correlated. For motif analysis, we extracted 100 bp flanking sequences from predicted peak summits and ran MEME for identifying statistically overrepresented motifs. We performed MAST to search motifs in the peaks using the model built by MEME.

\section{Identification of HOXC9 target genes}

Genes with HOXC9-binding peaks that are non-intergenic (i.e., within $-5 \sim+5 \mathrm{~kb}$ of genes) were defined as HOXC9 target genes. To correlate HOXC9 binding to gene expression, we combined the HOXC9 ChIP-seq data with the HOXC9 microarray data using in-house scripts to generate a list of the genes whose regulatory elements are bound by HOXC9 and whose expression levels are significantly changed $(\geq+1.5$ and $\leq-1.5$ fold, $P<0.01)$ as the result of HOXC9 induction. The significantly up- and down-regulated HOXC9 target genes were then subjected to gene annotation enrichment analysis with DAVID v6.7, GSEA, and IPA.

\section{Immunoprecipitation and mass spectrometric analyses}

$\mathrm{BE}(2)-\mathrm{C} /$ Tet-Off/myc-HOXC9 cells were cultured in the absence of doxycycline for 6 days and nuclear extracts were prepared following the Dignam protocol [68] except that buffer $\mathrm{C}$ contained $300 \mathrm{mM} \mathrm{NaCl}$. Extracts from $1 \times 10^{7}$ cells were incubated with Protein A/G beads (Invitrogen) coated with $4 \mu \mathrm{g}$ mouse anti-Myc tag (clone 4A6, Millipore) or mouse IgG for overnight at $4{ }^{\circ} \mathrm{C}$. The beads were washed 3 times with buffer $\mathrm{C}$ containing $150 \mathrm{mM} \mathrm{NaCl}$, dried in a SpeedVac, re-suspended in a buffer containing $8 \mathrm{M}$ urea, $5 \mathrm{mM}$ DTT and $100 \mathrm{mM}$ ammonium bicarbonate, and alkylated with $15 \mathrm{mM}$ iodoacetamide for 1 hour. After alkylation, unreacted iodoacetamide was removed by $15 \mathrm{mM}$ DTT and the urea concentration was diluted to $\sim 1 \mathrm{M}$ with a buffer containing $50 \mathrm{mM}$ ammonium bicarbonate and $2 \mathrm{mM} \mathrm{CaCl}_{2}$. Immunoprecipitated proteins were digested with $14 \mathrm{ng} / \mu \mathrm{l}$ sequencing grade trypsin (Promega) for 24 hours at $37^{\circ} \mathrm{C}$. The digests were desalted with a Micro Trap desalting cartridge (Michrom BioResources), and tryptic peptides eluted with LC-MS Solvent B (90/10/0.05\%: Acetonitrile/water/ heptafluorobutyric acid) and dried in a SpeedVac. The digests were analyzed by Nano-HPLC using a Nano Trap column (CL5/61241/00, Michrom BioResources) and an Agilent 1200 Series Nano pump (Agilent Technologies) equipped with a refrigerated autosampler. An Agilent 1200 Series Capillary LC loading pump was used to introduce the sample onto a Captrap cartridge for sample concentration and de-salting.

Data-dependent MS and MS/MS spectra were acquired on an LTQ Orbitrap Discovery (Thermo Fisher Scientific) using 2 micro-scans, with a maximum injection time of 200 ms with 2 Da peak isolation width. Six scan events were recorded for each data acquisition cycle. The first scan event, acquired by the FTMS, was used for full scan MS acquisition from $300-2000 \mathrm{~m} / \mathrm{z}$. Data were recorded in the Centroid mode only. The remaining five scan events were used for collisionally activated dissociation (CAD): the five most abundant ions in each peptide MS 
were selected and fragmented to produce product-ion mass spectra.

\section{Database searching and protein identification}

All MS/MS data were analyzed using BioWorks Rev.3.3.1 SP1 (Thermo Fisher Scientific) and X!Tandem (thegpm. org). SEQUEST was set up to search NCBInr_Homosapiens_05262011.fasta (221863 entries) and the human.protein_RefSeq_01192012 database (33376), and X!Tandem was set up to search subsets of the databases. SEQUEST and X!Tandem were searched with a fragment ion mass tolerance of $0.80 \mathrm{Da}$ and a parent ion tolerance of 10.0 PPM. Scaffold (Proteome Software) was used to validate MS/MS-based peptide and protein identifications. Peptide identifications were accepted if they could be established at greater than $95.0 \%$ probability as specified by the Peptide Prophet algorithm [69]. Protein identifications were accepted if they could be established at greater than 90.0\% probability and contained at least 1 identified peptide. Protein probabilities were assigned by the Protein Prophet algorithm [70]. Proteins that contained similar peptides and could not be differentiated based on MS/MS analysis alone were grouped to satisfy the principles of parsimony. Single-peptide protein identification was accepted only if the protein was independently identified by both SEQUEST and X!Tandem.

\section{Size-exclusion chromatography}

Size-exclusion chromatography was performed with a Superose-6 10/300 GL column (24 ml bed volume) and an AKTA purifier (GE Healthcare). Nuclear extracts $(0.5 \mathrm{ml})$ were loaded onto the column equilibrated with PBS, and $0.5 \mathrm{ml}$ fractions were collected and analyzed.

\section{Co-immunoprecipitation}

Nuclear extracts or pooled Sepharose-6 fractions were incubated with protein $\mathrm{A} / \mathrm{G}$ beads coated with mouse anti-Myc tag (clone 4A6), mouse anti-E2F6, or control mouse IgG for 2 hours at $4^{\circ} \mathrm{C}$. After washing 3 times with PBS, the beads were suspended in standard SDS sample buffer and analyzed by immunoblotting.

\section{Immunoblotting}

Unless indicated, all antibodies were from Santa Cruz Biotechnologies. Samples were suspended in SDS sample buffer and boiled. Proteins were separated on SDSpolyacrylamide gels, transferred to nitrocellulose membranes, and probed with the following primary antibodies: rabbit anti-cyclin A2 (sc-751, 1:200), rabbit anti-cyclin B1 (sc-752, 1:200), mouse anti-myc-tag (9E10, hybridoma supernatant, 1:10), rabbit anti-E2F6 (sc-22823, 1:200), mouse anti-MEIS2 (63-T, sc-81986, 1:400), mouse antiNEFM (NF-09, sc-51683, 1:200), and rabbit anti- $\beta$ actin (600-401-886, Rockland Immunochemicals, 1:2000).
Horseradish peroxidase-conjugated goat anti-mouse and goat anti-rabbit IgG were used as secondary antibodies. Proteins were visualized using a SuperSignal West Pico chemiluminescence kit (Pierce, Thermo Fisher Scientific) and quantified with ImageJ (National Institutes of Health). For visualization and quantification with the Odyssey system, goat anti-mouse IRDye 800 , anti-rabbit IRDye 800 , anti-mouse IRDye 680, and anti-rabbit IRDye 680 were used as secondary antibodies (LI-COR Biosciences).

\section{Statistics}

All quantitative data were analyzed and presented with GraphPad Prism 5.0f for Mac using unpaired, two-tailed Student's $t$-test.

\section{Additional files}

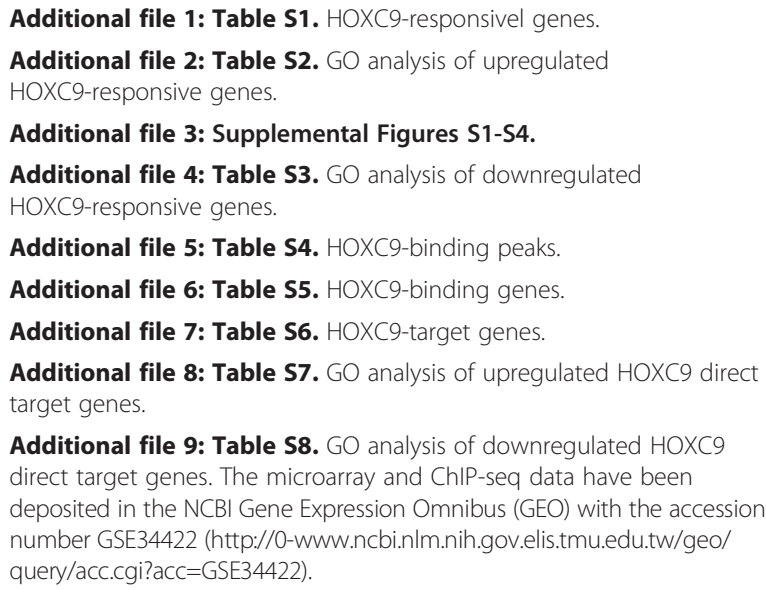

\section{Abbreviations}

BER: Base excision repair; CCN: Cyclin; CDK: Cyclin-dependent kinase; ChIP-seq: Chromatin immunoprecipitation and sequencing; Co-IP: Co-immunoprecipitation; DAVID: Database for annotation visualization and integrated discovery; DMEM: Dulbecco's modified eagle medium; DSB: Double-strand break; FA: Fanconi anemia; FDR: False discovery rate; FoxP1: Forkhead box protein P1; GSEA: Gene set enrichment analysis; HMC: Hypaxial motor column; IPA: Ingenuity pathways analysis; LC-MS: Liquid chromatography; Mass: Spectrometry; LMC: lateral motor column; MMR: Mismatch repair; NEFM: Neurofilament medium; NER: Nucleotide excision repair; PBS: Phosphate buffered saline; PGC: Preganglionic motor column; RA: Retinoic acid; TSS: Transcription start site; UTR: Untranslated region.

\section{Competing interests}

The authors declare that they have no competing interests.

\section{Authors' contributions}

$X W, J D, L Y$ and $H$-FD performed experiments with the assistance of $\mathrm{HC}$ and LM in establishing and characterizing inducible HOXC9 expression cells, LM in microarray sample preparation, BJ and MR in ChIP, EJL in ChIP-seq library preparation, and LCN in mass spectrometry. J-HC YZ, and H-FD performed microarray and ChIP-seq data analyses. XW, JD, HC, and H-FD designed the study with the assistance of $\mathrm{HS}, \mathrm{JC}$, and $\mathrm{SH} \mathrm{H}-\mathrm{FD}$ wrote the manuscript with contributions from JD, J-HC, LCN and EJL. All authors read and approved the final manuscript. 


\section{Acknowledgements}

We thank Drs. LesleyAnn Hawthorn and Sam Chang at the Georgia Regents University Cancer Center Genomics Core for assistance in Microarray and ChIP-seq. This work was supported by grants from the National Institutes of Health (CA124982) and Department of Defense (W81XWH-12-1-0613) to H.F. D., the National Basic Research Program of China (No. 2012cb114603) to H.C. and the National Natural Science Foundation of China (No. 81172443) to X. W. H.F.D., H.S., and J.C. are Georgia Cancer Coalition Distinguished Scholars.

\section{Author details}

'Department of Urology, Second Affiliated Hospital, Third Military Medical University, Chongqing, China. ${ }^{2}$ Cancer Center, Georgia Regents University, Augusta, GA 30912, USA. ${ }^{3}$ Department of Biostatistics and Epidemiology, Medical College of Georgia, Georgia Regents University, Augusta, GA 30912, USA. ${ }^{4}$ Department of Pathology, Medical College of Georgia, Georgia Regents University, Augusta, GA 30912, USA. ${ }^{5}$ Department of Biochemistry and Molecular Biology, Medical College of Georgia, Georgia Regents University, Augusta, GA 30912, USA. 'State Key Laboratory of Silkworm Genome Biology, Institute of Sericulture and System Biology, Southwest University, Chongqing, China. ${ }^{7}$ Department of Neurology, First Hospital of Yichang, Three Gorges University College of Medicine, Yichang, China. ${ }^{8}$ Department of Neurology, Union Hospital, Tongji Medical College, Huazhong University of Science and Technology, Wuhan, China.

Received: 26 July 2013 Accepted: 20 November 2013

Published: 25 November 2013

\section{References}

1. Nouspikel T, Hanawalt PC: DNA repair in terminally differentiated cells. DNA Repair (Amst) 2002, 1(1):59-75.

2. Simonatto $M$, Latella L, Puri PL: DNA damage and cellular differentiation: more questions than responses. J Cell Physiol 2007, 213(3):642-648.

3. Fortini P, Dogliotti E: Mechanisms of dealing with DNA damage in terminally differentiated cells. Mutat Res 2010, 685(1-2):38-44.

4. Reya T, Morrison SJ, Clarke MF, Weissman IL: Stem cells, cancer, and cancer stem cells. Nature 2001, 414(6859):105-111.

5. Clarke MF, Dick JE, Dirks PB, Eaves CJ, Jamieson CH, Jones DL, Visvader J, Weissman IL, Wahl GM: Cancer stem cells-perspectives on current status and future directions: AACR workshop on cancer stem cells. Cancer Res 2006, 66(19):9339-9344.

6. Visvader JE, Lindeman GJ: Cancer stem cells in solid tumours: accumulating evidence and unresolved questions. Nat Rev Cancer 2008, 8(10):755-768

7. Bao S, Wu Q, McLendon RE, Hao Y, Shi Q, Hjelmeland AB, Dewhirst MW, Bigner $\mathrm{DD}$, Rich JN: Glioma stem cells promote radioresistance by preferential activation of the DNA damage response. Nature 2006, 444(7120):756-760,

8. Phillips TM, McBride WH, Pajonk F: The response of CD24(-/low)/CD44+ breast cancer-initiating cells to radiation. J Natl Cancer Inst 2006, 98(24):1777-1785.

9. Eramo A, Ricci-Vitiani L, Zeuner A, Pallini R, Lotti F, Sette G, Pilozzi E, Larocca LM, Peschle C, De Maria R: Chemotherapy resistance of glioblastoma stem cells. Cell Death Differ 2006, 13(7):1238-1241.

10. Liu G, Yuan X, Zeng Z, Tunici P, Ng H, Abdulkadir IR, Lu L, Irvin D, Black KL, YU JS: Analysis of gene expression and chemoresistance of CD133+ cancer stem cells in glioblastoma. Mol Cancer 2006, 5:67.

11. Pearson JC, Lemons D, McGinnis W: Modulating Hox gene functions during animal body patterning. Nat Rev Genet 2005, 6(12):893-904.

12. Moens CB, Selleri L: Hox cofactors in vertebrate development. Dev Biol 2006, 291(2):193-206.

13. Shah N, Sukumar S: The Hox genes and their roles in oncogenesis. Nat Rev Cancer 2010, 10(5):361-371.

14. Argiropoulos B, Humphries RK: Hox genes in hematopoiesis and leukemogenesis. Oncogene 2007, 26(47):6766-6776.

15. Brodeur GM: Neuroblastoma: biological insights into a clinical enigma. Nat Rev Cancer 2003, 3:203-216.

16. Maris JM: Recent advances in neuroblastoma. N Engl J Med 2010 362(23):2202-2211.

17. Kocak H, Ackermann $S$, Hero B, Kahlert $Y$, Oberthuer A, Juraeva D, Roels F, Theissen J, Westermann F, Deubzer $\mathrm{H}$, et al: Hox-C9 activates the intrinsic pathway of apoptosis and is associated with spontaneous regression in neuroblastoma. Cell Death Dis 2013, 4:e586.
18. Mao L, Ding J, Zha Y, Yang L, McCarthy BA, King W, Cui H, Ding HF: HOXC9 Links cell-cycle exit and neuronal differentiation and is a prognostic marker in neuroblastoma. Cancer Res 2011, 71(12):4314-4324.

19. Seeds NW, Gilman AG, Amano T, Nirenberg MW: Regulation of axon formation by clonal lines of a neural tumor. Proc Natl Acad Sci USA 1970, 66(1):160-167.

20. Jensen L, Linn S: A reduced rate of bulky DNA adduct removal is coincident with differentiation of human neuroblastoma cells induced by nerve growth factor. Mol Cell Biol 1988, 8(9):3964-3968.

21. Sidell N: Retinoic acid-induced growth inhibition and morphologic differentiation of human neuroblastoma cells in vitro. J Natl Cancer Inst 1982, 68(4):589-596.

22. Abemayor $\mathrm{E}$, Sidell $\mathrm{N}$ : Human neuroblastoma cell lines as models for the in vitro study of neoplastic and neuronal cell differentiation. Environ Health Perspect 1989, 80:3-15.

23. Zha Y, Ding E, Yang L, Mao L, Wang X, McCarthy BA, Huang S, Ding HF: Functional dissection of HOXD cluster genes in regulation of neuroblastoma cell proliferation and differentiation. PLOS One 2012, 7(8):e40728.

24. Byfield JE, Lee YC, Klisak I, Finklestein JZ: Effect of differentiation on the repair of DNA single strand breaks in neuroblastoma cells. Biochem Biophys Res Commun 1975, 63(3):730-735.

25. McCombe P, Lavin M, Kidson C: Control of DNA repair linked to neuroblastoma differentiation. Int J Radiat Biol 1976, 29(6):523-531.

26. Lavin MF, McCombe P, Kidson C: DNA replication and post-replication repair in U.V.-sensitive mouse neuroblastoma cells. Int J Radiat Biol 1976, 30(1):31-40.

27. James M, Mansbridge J, Kidson C: Ultraviolet radiation sensitivity of proliferating and differentiated human neuroblastoma cells. Int J Radiat Biol 1982, 41(5):547-556.

28. Duester $\mathrm{G}$ : Retinoic acid synthesis and signaling during early organogenesis. Cell 2008, 134(6):921-931.

29. Dennis G Jr, Sherman BT, Hosack DA, Yang J, Gao W, Lane HC, Lempicki RA: DAVID: Database for Annotation, Visualization, and Integrated Discovery. Genome Biol 2003, 4(5):3.

30. Huang DW, Sherman BT, Lempicki RA: Systematic and integrative analysis of large gene lists using DAVID bioinformatics resources. Nat Protoc 2008, 4(1):44-57.

31. Francis NJ, Landis SC: Cellular and molecular determinants of sympathetic neuron development. Annu Rev Neurosci 1999, 22:541-566.

32. Goridis C, Rohrer H: Specification of catecholaminergic and serotonergic neurons. Nat Rev Neurosci 2002, 3(7):531-541.

33. Ernsberger U: The role of GDNF family ligand signalling in the differentiation of sympathetic and dorsal root ganglion neurons. Cell Tissue Res 2008, 333(3):353-371.

34. Rajasekharan S, Kennedy T: The netrin protein family. Genome Biol 2009, 10(9):239.

35. Jung H, Lacombe J, Mazzoni EO, Liem KF Jr, Grinstein J, Mahony S, Mukhopadhyay D, Gifford DK, Young RA, Anderson KV, et al: Global control of motor neuron topography mediated by the repressive actions of a single hox gene. Neuron 2010, 67(5):781-796.

36. Bell SP, Dutta A: DNA replication in eukaryotic cells. Annu Rev Biochem 2002, 71(1):333-374.

37. Carmena M, Wheelock M, Funabiki H, Earnshaw WC: The chromosomal passenger complex (CPC): from easy rider to the godfather of mitosis. Nat Rev Mol Cell Biol 2012, 13(12):789-803.

38. Kee Y, D'Andrea AD: Expanded roles of the Fanconi anemia pathway in preserving genomic stability. Genes Dev 2010, 24(16):1680-1694.

39. Liu Y, Kao H-I, Bambara RA: FLAP ENDONUCLEASE 1: a central component of DNA metabolism. Annu Rev Biochem 2004, 73(1):589-615.

40. Trimarchi JM, Lees JA: Sibling rivalry in the E2F family. Nat Rev Mol Cell Biol 2002, 3(1):11-20.

41. Xu X, Bieda M, Jin VX, Rabinovich A, Oberley MJ, Green R, Farnham PJ: A comprehensive ChIP-chip analysis of E2F1, E2F4, and E2F6 in normal and tumor cells reveals interchangeable roles of E2F family members. Genome Res 2007, 17(11):1550-1561.

42. Dasen JS, Liu JP, Jessell TM: Motor neuron columnar fate imposed by sequential phases of Hox-c activity. Nature 2003, 425(6961):926-933.

43. Rousso DL, Gaber ZB, Wellik D, Morrisey EE, Novitch BG: Coordinated actions of the forkhead protein Foxp1 and Hox proteins in the columnar organization of spinal motor neurons. Neuron 2008, 59(2):226-240. 
44. Dasen JS, De Camilli A, Wang B, Tucker PW, Jessell TM: Hox repertoires for motor neuron diversity and connectivity gated by a single accessory factor, FoxP1. Cell 2008, 134(2):304-316

45. Edlund T, Jessell TM: Progression from extrinsic to intrinsic signaling in cell fate specification: a view from the nervous system. Cell 1999, 96(2):211-224

46. Ohnuma S, Harris WA: Neurogenesis and the cell cycle. Neuron 2003 40(2):199-208

47. Galderisi U, Jori FP, Giordano A: Cell cycle regulation and neural differentiation. Oncogene 2003, 22(33):5208-5219.

48. Salomoni $\mathrm{P}$, Calegari F: Cell cycle control of mammalian neural stem cells: putting a speed limit on G1. Trends Cell Biol 2010, 20(5):233-243.

49. Sherr CJ, Roberts JM: CDK inhibitors: positive and negative regulators of G1-phase progression. Genes Dev 1999, 13(12):1501-1512.

50. Ohnuma S, Philpott A, Wang K, Holt CE, Harris WA: p27Xic1, a Cdk inhibitor, promotes the determination of glial cells in Xenopus retina. Cell 1999, 99(5):499-510.

51. Nguyen L, Besson A, Heng Jl, Schuurmans C, Teboul L, Parras C, Philpott A, Roberts JM, Guillemot F: p27kip1 independently promotes neuronal differentiation and migration in the cerebral cortex. Genes Dev 2006 20(11):1511-1524.

52. Nieto M, Schuurmans C, Britz O, Guillemot F: Neural bHLH genes control the neuronal versus glial fate decision in cortical progenitors. Neuron 2001, 29(2):401-413.

53. Sukhanova MJ, Deb DK, Gordon GM, Matakatsu MT, Du W: Proneural basic helix-loop-helix proteins and epidermal growth factor receptor signaling coordinately regulate cell type specification and cdk inhibitor expression during development. Mol Cell Biol 2007, 27(8):2987-2996.

54. Morkel M, Wenkel J, Bannister AJ, Kouzarides T, Hagemeier C: An E2F-like repressor of transcription. Nature 1997, 390(6660):567-568

55. Gaubatz S, Wood JG, Livingston DM: Unusual proliferation arrest and transcriptional control properties of a newly discovered E2F family member, E2F-6. Proc Natl Acad Sci USA 1998, 95(16):9190-9195.

56. Cartwright $\mathrm{P}$, Muller $\mathrm{H}$, Wagener $\mathrm{C}$, Holm K, Helin K: E2F-6: a novel member of the E2F family is an inhibitor of E2F-dependent transcription. Oncogene 1998, 17(5):611-623.

57. Trimarchi JM, Fairchild B, Verona R, Moberg K, Andon N, Lees JA: E2F-6, a member of the E2F family that can behave as a transcriptional repressor. Proc Natl Acad Sci USA 1998, 95(6):2850-2855.

58. Giangrande PH, Zhu W, Schlisio S, Sun X, Mori S, Gaubatz S, Nevins JR: A role for E2F6 in distinguishing G1/S- and G2/M-specific transcription. Genes Dev 2004, 18(23):2941-2951.

59. Velasco G, Hube F, Rollin J, Neuillet D, Philippe C, Bouzinba-Segard H, Galvani A Viegas-Pequignot E, Francastel C: Dnmt3b recruitment through E2F6 transcriptional repressor mediates germ-line gene silencing in murine somatic tissues. Proc Natl Acad Sci USA 2010, 107(20):9281-9286.

60. Ogawa H, Ishiguro K, Gaubatz S, Livingston DM, Nakatani Y: A complex with chromatin modifiers that occupies E2F- and Myc-responsive genes in G0 cells. Science 2002, 296(5570):1132-1136.

61. Attwooll C, Oddi S, Cartwright P, Prosperini E, Agger K, Steensgaard P, Wagener C, Sardet C, Moroni MC, Helin K: A novel repressive E2F6 complex containing the polycomb group protein, EPC1, that interacts with EZH2 in a proliferation-specific manner. J Biol Chem 2005, 280(2):1199-1208

62. Deshpande AM, Akunowicz JD, Reveles XT, Patel BB, Saria EA, Gorlick RG, Naylor SL, Leach RJ, Hansen MF: PHC3, a component of the hPRC-H complex, associates with E2F6 during G0 and is lost in osteosarcoma tumors. Oncogene 2007, 26(12):1714-1722.

63. Trojer P, Cao AR, Gao Z, Li Y, Zhang J, Xu X, Li G, Losson R, Erdjument-Bromage H, Tempst $P$, et al: L3MBTL2 protein acts in concert with PcG protein-mediated monoubiquitination of $\mathrm{H} 2 \mathrm{~A}$ to establish a repressive chromatin structure. Mol Cell 2011, 42(4):438-450.

64. Nouspikel T, Hanawalt PC: Impaired nucleotide excision repair upon macrophage differentiation is corrected by E1 ubiquitin-activating enzyme. Proc Natl Acad Sci USA 2006, 103(44):16188-16193.

65. Subramanian A, Tamayo P, Mootha VK, Mukherjee S, Ebert BL, Gillette MA, Paulovich A, Pomeroy SL, Golub TR, Lander ES, et al: Gene set enrichment analysis: a knowledge-based approach for interpreting genome-wide expression profiles. Proc Natl Acad Sci USA 2005, 102(43):15545-15550.

66. Lee TI, Johnstone SE, Young RA: Chromatin immunoprecipitation and microarray-based analysis of protein location. Nat Protoc 2006, 1(2):729-748.
67. Zhang Y, Liu T, Meyer CA, Eeckhoute J, Johnson DS, Bernstein BE, Nusbaum C, Myers RM, Brown M, Li W, et al: Model-based analysis of ChIP-Seq (MACS). Genome Biol 2008, 9(9):R137

68. Dignam JD, Lebovitz RM, Roeder RG: Accurate transcription initiation by RNA polymerase II in a soluble extract from isolated mammalian nuclei. Nucleic Acids Res 1983, 11(5):1475-1489.

69. Keller A, Nesvizhskii Al, Kolker E, Aebersold R: Empirical statistical model to estimate the accuracy of peptide identifications made by MS/MS and database search. Anal Chem 2002, 74(20):5383-5392.

70. Nesvizhskii Al, Keller A, Kolker E, Aebersold R: A statistical model for identifying proteins by tandem mass spectrometry. Anal Chem 2003, 75(17):4646-4658.

\section{doi:10.1186/1471-2164-14-830}

Cite this article as: Wang et al:: HOXC9 directly regulates distinct sets of genes to coordinate diverse cellular processes during neuronal differentiation. BMC Genomics 2013 14:830.

\section{Submit your next manuscript to BioMed Central and take full advantage of:}

- Convenient online submission

- Thorough peer review

- No space constraints or color figure charges

- Immediate publication on acceptance

- Inclusion in PubMed, CAS, Scopus and Google Scholar

- Research which is freely available for redistribution
C BioMed Central 